\title{
Evaluación de una experiencia de capacitación en planificación educacional para directores de programas de las especialidades médicas
}

\author{
MARISOL SIRHAN, XIMENA TRIVIÑO
}

Centro de Educación Médica, Escuela de Medicina, Pontificia Universidad Católica de Chile, Santiago de Chile.

Recibido el 13 de junio de 2011, aceptado el 15 de diciembre de 2011.

Correspondencia a: Dra. Marisol Sirhan Nahum Centro de Educación Médica, Escuela de Medicina. Pontificia Universidad Católica de Chile.

Lira 44, Santiago Centro, Santiago, Chile. Tel.: (56-2) 3543476 Fax: (56-2) 6322802 Correo electrónico: msirhan@med.puc.cl

\section{Evaluation of a faculty development program in curriculum development for program directors of medical specialties}

Background: Few medical teaching institutions provide faculty development in curriculum development to program directors of medical specialties $(P D)$, despite the increased demand for renewal of residency programs and the evaluation of outcomes. Aim: To describe and evaluate a training program in curriculum development for PD developed in 2008 and 2009. Material and Methods: Thirty PD attended an on campus course of fifteen hours. Evaluation was done using Kirkpatrick model through an end-of-course questionnaire, a retrospective pre/post self-assessment test of skills, the assessment of learning and the final projects developed by PD. Results: All PD finished the course and answered the questionnaire. In level 1 (Reaction), 100\% reported high satisfaction and would recommend it highly to others, with perceived mean achievement of course objectives of $81 \%$. In level 2 (Learning), all the differences between the retrospective pre and posttest were statistically significant $(p<0.01)$, and achievement of learning was in average $82.9 \%$. In level 3 (Behavior), 100\% felt they would apply what was learnt and 17 PD (57\%) sent projects. Conclusions: This model of faculty development was highly accepted by PD and had a positive evaluation based in high satisfaction, the improvement in pre/posttest assessment, the achievement of learning objectives and the development of projects.

(Rev Med Chile 2012; 140: 530-537).

Key words: Education, medical, graduate; Curriculum; Faculty, medical.
$\mathrm{E}$ n el último decenio los cuerpos colegiados de las especialidades médicas del postítulo de Estados Unidos de Norteamérica, Canadá y Reino Unido han impulsado proyectos de reformulación y perfeccionamiento de sus programas de formación de especialistas, con un enfoque basado en competencias y en la evaluación de resultados ${ }^{1-4}$. Con esta aproximación se espera dar respuesta a las demandas de la sociedad para cautelar aspectos fundamentales como garantizar una formación profesional competente y responsable en todas las especialidades médicas, profundizar la enseñanza en temas de gestión, prevención y promoción de la salud, además de brindar una atención segura y de calidad en el cuidado de los pacientes ${ }^{5}$.

Los directores de los programas de postítulo de las especialidades médicas (DPP) que lideran la formación de especialistas como expertos en sus disciplinas, requieren una formación en docencia para implementar estos cambios y lograr su perfeccionamiento continuo 6 . Además de la experiencia en la dirección del programa, es necesario dominar los fundamentos pedagógicos para el aprendizaje, 
manejar metodologías efectivas, evaluar las competencias en la práctica y desarrollar habilidades en planificación y gestión educacional ${ }^{7-13}$. Todos estos aspectos han sido identificados como deficientes por los DPP, constituyéndose en limitaciones para la conducción de los programas y en consecuencia en prioridades de capacitación ${ }^{14-17}$.

Aunque en la literatura se describe una amplia gama de cursos y programas de formación en docencia para los profesores de Medicina, sólo un escaso número se han implementado para capacitar a los DPP y han sido creados por centros formadores universitarios o sociedades de especialistas bajo las directrices de organizaciones centralizadas como CanMeds y ACGME, sin considerar las necesidades específicas referidas por los $\mathrm{DPP} \mathrm{P}^{4,18}$, 19. Más aún, en la mayoría de estos programas la capacitación en planificación educacional ha sido parcial y con una profundidad e intensidad variable ${ }^{20,21}$. En 2008, destacó el estudio de Kern y colaboradores sobre los resultados a largo plazo de un programa de capacitación longitudinal en desarrollo curricular para docentes clínicos que tuvo un impacto positivo en la adquisición de conocimientos y competencias relacionadas, así como en la implementación de los nuevos programas que desarrollaron los docentes con efecto perdurable en el tiempo ${ }^{22}$.

\section{¿Qué ha sucedido con los DPP de la Escuela de Medicina de la Pontificia Universidad Católica de Chile (EMUC)?}

A pesar de que desde el 2000 se comenzó a dictar el Diplomado en Educación Médica para los docentes de la EMUC, al 2007 sólo siete DPP habían completado el diploma y 46 (89\%) DPP no habían realizado ninguno de los cursos del programa $^{23}$. Por ello, ese año la dirección de la EMUC propuso desarrollar una capacitación formal en docencia dirigida a los DPP de las especialidades y subespecialidades para profesionalizar la docencia, perfeccionar los programas y facilitar los procesos de acreditación. En 2008 y 2009 se implementó un programa de capacitación longitudinal adaptado para los DPP de 180 horas, con cursos secuenciales y de modalidad presencial. Uno de los cursos del programa para DPP fue "Desarrollo Curricular para Directores de Programas de Postítulo en Especialidades Médicas".

El objetivo del estudio fue describir y evaluar una experiencia de capacitación en planificación educacional para los DPP con el curso "Desarrollo Curricular para Directores de Programas de Postítulo en Especialidades Médicas" realizado en la EMUC en 2008 y 2009.

\section{Material y Método}

Estudio descriptivo, retrospectivo y transversal.

\section{Descripción del programa}

Un mes antes de iniciar el curso se aplicó una encuesta a un grupo de DPP de la EMUC para determinar sus necesidades de capacitación $(\mathrm{n}=17)$. Doce $(71 \%)$ manifestaron necesitar capacitación en temas curriculares y evaluación de programas, a pesar de que nueve (53\%) reportaron haber recibido algún entrenamiento en diseño de cursos y 11 (64\%) en evaluación de la docencia. La mayoría se manifestó interesado en perfeccionar su programa, evaluarlo y facilitar la profesionalización de la docencia en el postítulo. Otros intereses expresados espontáneamente fueron "compartir experiencias" e "identificar soluciones a problemas comunes de los programas".

Con esta información se completó el diseño del programa utilizando el modelo en seis etapas de Kern ${ }^{24}$. Esta aproximación sistemática e integral propone una vez definido el problema y el contexto (etapa 1), situar en el centro las necesidades de aprendizaje de los participantes (etapa 2), para alcanzar un propósito con objetivos definidos (etapa 3), con énfasis en el uso de metodologías que promuevan un aprendizaje activo y auto dirigido (etapa 4). El siguiente paso consiste en planificar la implementación (etapa 5) y finalmente, evaluar los aprendizajes y el programa para objetivar el impacto educacional (etapa 6).

Dado que las estrategias y los métodos empleados son tan relevantes como los contenidos para lograr un aprendizaje efectivo, en este curso para DPP además de seguir los principios de educación de adultos de Knowles, se propuso la práctica reflexiva mientras se aprende y sobre lo aprendido formulada por Schon, el aprender practicando y la revisión de las prácticas docentes considerando presunciones, creencias personales y la autocrítica contrastada con otras fuentes de credibilidad, propuestos por Harris y Armstrong respectivamente ${ }^{25-27}$. Más aún, se facilitó la reflexión en escenarios realistas y cercanos a los roles docentes para promover la enseñanza efectiva y mejorar la 
docencia de modo que los aprendizajes fueran transferibles y aplicables a la práctica ${ }^{28}$.

Los objetivos de aprendizaje fueron: aplicar los modelos de Kern y Kirkpatrick en el diseño y evaluación de cursos y programas, ejercitar habilidades en planificación y gestión educacional, desarrollar un portafolio de práctica reflexiva, elaborar un proyecto de intervención en el programa de la especialidad, revisar metodologías de análisis curricular para la implementación de cambios y reflexionar sobre desafíos e innovaciones curriculares en el postítulo. Los contenidos del curso se muestran en la Tabla 1.

Las metodologías empleadas fueron: estudio

\section{Tabla 1. Contenidos de la capacitación en} planificación educacional

\footnotetext{
Marco teórico y fundamentos del desarrollo curricular en Medicina

- De deliberación

- De reformulación

Gestión educacional

- Planificación con enfoque por competencias

- Metas del aprendizaje y desempeños trazadores

- Componentes básicos de las competencias (descripción, niveles, escala, condiciones de medición y criterios)

- Sistema de evaluación del aprendizaje y adquisición de competencias según niveles de la pirámide de Miller

Diseño de cursos y programas según Kern

- Identificar el problema y realizar una evaluación general de necesidades

- Evaluar las necesidades de aprendizaje de los estudiantes

- Definir propósito y objetivos

- Seleccionar estrategias y metodologías

- Planificar la implementación

- Evaluación de aprendizajes y evaluación del programa

Modelo de evaluación de programas educacionales según Kirkpatrick

- Nivel 1: Reacción

- Nivel 2: Aprendizaje

- Nivel 3: Conducta

- Nivel 4: Resultados

Temas curriculares emergentes en el Postítulo de Medicina

- Acreditación de programas de postítulo

- Certificación del especialista

- Aprendizaje y entrenamiento de competencias

- Evaluación de procedimientos
}

personal, discusión, análisis de escenarios educacionales, trabajo en pequeño grupo, lecturas, clases interactivas, portafolio, debate por equipos y desarrollo de un proyecto educacional con retroalimentación. El portafolio se usó para ejercitar la práctica reflexiva y recolectar el trabajo personal basado en: la autoevaluación del programa de la especialidad, fortalezas y debilidades del DPP, análisis de la encuesta de evaluación periódica de la docencia, definición de competencias y plan de evaluación de desempeños trazadores ${ }^{29,30}$. El debate, una metodología novedosa en educación médica, permitió ejercitar la capacidad de síntesis, la lectura crítica basada en la evidencia y la búsqueda eficiente de información en revistas educacionales $^{31}$. Los temas seleccionados fueron pertinentes al postítulo: certificación del especialista por el centro formador versus agencia externa, entrenamiento con pacientes reales versus pacientes simulados, y evaluación de procedimientos en situaciones clínicas reales versus situaciones simulada (Tabla 2). Con el propósito de consolidar lo aprendido y facilitar su aplicación a la práctica docente cada DPP debió desarrollar un el proyecto de intervención en el programa de la especialidad.

Para evaluar los aprendizajes se utilizaron pautas de autoevaluación, evaluación por pares, revisión del portafolio, desempeño en el debate, proyecto de intervención y apreciación global del desempeño. Las escalas usadas fueron de cotejo (sí/no), apreciación (excelente, muy bueno, bueno, límite, insuficiente) y de desempeño (logrado / no logrado) (Tabla 3 ).

El curso se realizó en seis sesiones semanales de 2,5 horas (duración 15 horas), con modalidad presencial y fue facilitado por el director de curso $y$ un docente.

\section{Sujetos}

Realizaron el curso 30 DPP, 17 el 2008 y 13 el 2009 , que representaban el $65 \%$ de los docentes que no habían recibido capacitación. Fueron 20 (66,6\%) hombres, y en su mayoría eran Profesores Titulares y Asociados, pertenecientes a las siguientes especialidades: Medicina Interna, Reumatología, Nefrología, Cardiología Intervencional, Medicina Intensiva, Medicina de Urgencia, Psiquiatría, Pediatría, Infectología Pediátrica, Cardiología Pediátrica, Enfermedades Respiratorias del niño, Medicina Intensiva Pediátrica, HematoOncología Pediátrica, Obstetricia y Ginecología, 
EDUCACIÓN MÉDICA

Capacitación en planificación educacional para directores de programas de especialidades - M. Sirhan et al

Tabla 2. Formato del debate por equipos*

\begin{tabular}{|llccc|}
\hline Sección & Desarrollo & $\begin{array}{c}\text { Tiempo } \\
\text { Equipo A } \\
\text { (minutos) }\end{array}$ & $\begin{array}{c}\text { Tiempo } \\
\text { Equipo B } \\
\text { (minutos) }\end{array}$ & $\begin{array}{c}\text { Tiempo } \\
\text { total } \\
\text { (minutos) }\end{array}$ \\
\hline I: Justificación de la defensa & Argumento: Introducción, fortalezas y usos & 4 & 4 & 8 \\
\hline II: Ataque al oponente & Contraargumento: Debilidades y dificultades & 3 & 3 & 6 \\
\hline III: Preguntas al oponente & Pregunta abierta: 1 por equipo & Respuesta: 1,5 & Respuesta: 1,5 & 3 \\
\hline IV: Cierre & Defensa final & 2 & 2 & 4 \\
V: Retroalimentación & Audiencia: Comentarios y retroalimentación & 4 & 4 & 8 \\
\hline
\end{tabular}

* No está permitido usar medios audiovisuales. La reacción y la reflexión de los asistentes (audiencia) son fundamentales. Los asistentes deben estar listos para opinar críticamente con fundamentos.

Tabla 3. Pauta de desempeño en el debate por equipos

\begin{tabular}{|c|c|c|c|c|c|}
\hline Criterios de evaluación & Excelente & Muy Bueno & Bueno & Regular & Insuficiente \\
\hline \multicolumn{6}{|c|}{ Relevancia y dominio de los argumentos a favor } \\
\hline \multicolumn{6}{|c|}{ Claridad y efectividad de la crítica al otro equipo } \\
\hline \multicolumn{6}{|l|}{ Interés y originalidad de la pregunta } \\
\hline \multicolumn{6}{|l|}{ Precisión y seguridad de la respuesta } \\
\hline \multicolumn{6}{|l|}{ Calidad de la defensa final y cierre } \\
\hline Coherencia global de la presentación & & & & & \\
\hline
\end{tabular}

Cirugía, Cirugía Digestiva, Cirugía Vascular, Urología, Neurocirugía, Cirugía Infantil, Ortopedia y Traumatología, Oftalmología, Dermatología, Radiología, Medicina Nuclear, Radioncología, y Magíster de Bioética.

\section{Evaluación de la intervención}

Para determinar el impacto de la capacitación se utilizó el modelo de evaluación Kirkpatrick que ha sido propuesto para intervenciones educacionales y explora efectos en cuatro niveles: satisfacción de los participantes con el programa, organización, contenidos, métodos, profesores y experiencia de aprendizaje (Nivel 1 Reacción), adquisición de conocimientos y destrezas y/o cambio en las percepciones hacia lo aprendido (Nivel 2 Aprendizaje), disposición para aplicar los conocimientos y destrezas y/o a practicar lo aprendido en el lugar de trabajo (Nivel 3 Conducta) y cambios en la organización y/o en el aprendizaje de estudiantes /residentes como consecuencia del programa (Nivel 4 Resultados) $)^{32-34}$.

Se utilizó una encuesta anónima y semiestructurada para explorar los tres primeros niveles de Kirkpatrick que fue aplicada a todos los DPP al finalizar el curso. Se incluyó un cuestionario prepost retrospectivo sobre percepción de habilidades en planificación educacional presentes al inicio y al finalizar la capacitación con una escala de Likert de 5 puntos como evidencia para el Nivel $2^{35}$. Los aprendizajes evaluados fueron usados como fuente de información para el Nivel 2 y los proyectos de intervención recolectados como evidencia para el Nivel 3. El Nivel 4 no fue explorado.

\section{Análisis estadístico}

Los resultados fueron analizados con el programa Statistical Package for Social Sciences (SPSS versión 11.0). Se usó el test de Wilcoxon para analizar las diferencias en los indicadores del cuestionario pre-post retrospectivo y se consideró estadísticamente significativo un valor de $\mathrm{p}<0,05$. 


\section{Resultados}

Aprobaron el curso de capacitación y respondieron la encuesta de evaluación los 30 (100\%) DPP. A continuación se presentan los resultados de la evaluación agrupados por niveles según el modelo de Kirkpatrick.

\section{Nivel 1 Reacción}

Respecto de la satisfacción global con el programa y la organización del curso, todos los DPP (100\%) lo consideraron interesante, útil, organizado, con metodologías adecuadas y que lo recomendarían a otros. En relación a los profesores, 28 (94\%) manifestaron que facilitaron el aprendizaje, explicaron con claridad, mantuvieron el interés y entregaron retroalimentación constructiva. Los contenidos y el tiempo asignado a las actividades fueron considerados adecuados por $25(84 \%)$ y $22(74 \%)$ respectivamente. El porcentaje promedio percibido de cumplimiento de los objetivos fue $81 \%$. Los aspectos más destacados fueron el ambiente de aprendizaje, el estilo participativo, el trabajo en pequeño grupo, los debates y la aplicabilidad de las tareas a la práctica docente. Mencionaron espontáneamente "todo, especialmente darme cuenta del profesionalismo con que trabajan", "la dedicación y empeño en formar al cuerpo docente", "tocar aspectos críticos en mi programa", "la posibilidad de mejorar mi programa", "el intercambio de opiniones con otros jefes de programa”.

Para perfeccionarlo recomendaron aumentar el número de sesiones, extender el tiempo de discusión y reducir las lecturas en número y complejidad.

\section{Nivel 2 Aprendizaje}

En relación a los aprendizajes evaluados, el rendimiento final promedio fue $82,9 \%$ (rango $65,7-97,1 \%)$. Los resultados desglosados por instrumento fueron: autoevaluación 90\% (rango: 71,4\%-100\%), evaluación por pares $94,3 \%$ (rango: $81,4-100 \%$ ), portafolio $95,7 \%$ (rango: $84,3-100 \%$ ), debate por equipos $80 \%$ (rango: $72,9-90 \%$ ), proyecto de intervención 94,3\% (rango: 85,7-100\%) y apreciación global del desempeño $87,1 \%$ (rango: 71,4-100\%). Basados en los resultados de la encuesta $14(47 \%)$ DPP reportaron que habían aprendido lo esperado y 16 (53\%) más de lo esperado. Los resultados del pre post test retrospectivo sobre habilidades en planificación educacional se muestran en la Tabla 4. Los valores para todos los indicadores del post-test retrospectivo fueron superiores a los del pre-test retrospectivo y sus diferencias fueron estadísticamente significativas (test de Wilcoxon $\mathrm{p}<0,005$ ).

Tabla 4. Evaluación pre-post retrospectiva de la adquisición de habilidades docentes. $\mathbf{N}=13$

\begin{tabular}{|c|c|c|c|c|c|c|}
\hline Habilidades docentes & & Promedio & DS & Mínimo & Máximo & Test Wilcoxon \\
\hline \multirow[t]{2}{*}{ Planificar una experiencia educativa } & Pre & 2,1 & 0,4 & 2 & 3 & $0,001 *$ \\
\hline & Post & 3,6 & 0,5 & 3 & 4 & \\
\hline \multirow[t]{2}{*}{ Diseñar un curso } & Pre & 1,9 & 0,8 & 1 & 3 & $0,001 *$ \\
\hline & Post & 3,1 & 0,6 & 2 & 4 & \\
\hline \multirow[t]{2}{*}{ Escribir objetivos de aprendizaje } & Pre & 2,2 & 0,7 & 1 & 3 & $0,003^{*}$ \\
\hline & Post & 3,6 & 0,5 & 3 & 4 & \\
\hline \multirow[t]{2}{*}{ Seleccionar metodologías } & Pre & 2 & 0,7 & 1 & 3 & $0,001 *$ \\
\hline & Post & 3,5 & 0,7 & 2 & 4 & \\
\hline \multirow{2}{*}{$\begin{array}{l}\text { Planificar un sistema de evaluación de } \\
\text { aprendizajes }\end{array}$} & Pre & 2 & 0,7 & 1 & 3 & $0,002^{*}$ \\
\hline & Post & 3,5 & 0,9 & 1 & 4 & \\
\hline \multirow[t]{2}{*}{ Evaluar cursos/Programas } & Pre & 2 & 0,8 & 1 & 3 & $0,002^{*}$ \\
\hline & Post & 3,3 & 0,6 & 2 & 4 & \\
\hline
\end{tabular}

${ }^{*} \mathrm{p}<0,005$. Escala de habilidades docentes: $1=$ ninguna; 2 = escasa; $3=$ intermedio; 4 = avanzado (competente); $5=$ experto. 
EDUCACIÓN MÉDICA

Capacitación en planificación educacional para directores de programas de especialidades - M. Sirhan et al

Tabla 5. Proyectos de intervención en el programa de la especialidad presentados por los directores de programas de postítulo

\begin{tabular}{|c|c|}
\hline Área & Título del proyecto \\
\hline \multirow{5}{*}{$\begin{array}{l}\text { Elaboración de } \\
\text { programas }\end{array}$} & Nuevo programa de postítulo en Cardiología Pediátrica \\
\hline & Nuevo programa de postítulo en Hemato-Oncología Pediátrica \\
\hline & Nuevo curso de Cirugía Pediátrica para residentes de Pediatría y Medicina Familiar del Niño \\
\hline & Curso de nivelación para residentes al ingreso del programa de Pediatría \\
\hline & Reformulación del módulo Laboratorio de Virología para los programas de postítulo pediátricos \\
\hline \multirow{3}{*}{$\begin{array}{l}\text { Planificación por } \\
\text { competencias }\end{array}$} & Planificación para el programa de postítulo en Dermatología basado en competencias \\
\hline & Planificación para el programa de postítulo en Medicina Intensiva Pediátrica basado en competencias \\
\hline & Plan para incorporar competencias específicas en el programa de postítulo en Medicina Intensiva \\
\hline \multirow[t]{2}{*}{ Metodologías } & $\begin{array}{l}\text { Metodología y evaluación para el razonamiento clínico y toma de decisiones en el programa de } \\
\text { postítulo en Radioncología }\end{array}$ \\
\hline & Enseñanza y evaluación de procedimientos en el programa de postítulo en Neurocirugía \\
\hline \multirow{6}{*}{$\begin{array}{l}\text { Evaluación de } \\
\text { aprendizajes }\end{array}$} & Evaluación formativa de destrezas quirúrgicas en el programa de postítulo en Cirugía Vascular \\
\hline & Plan de evaluación de competencias generales en el programa de postítulo en Infectología Pediátrica \\
\hline & Evaluación de competencias generales en el programa de postítulo en Reumatología \\
\hline & Evaluación de actitudes y destrezas psicomotoras en el programa de postítulo en Dermatología \\
\hline & Plan de evaluación de procedimientos invasivos en el programa de postítulo de Medicina Interna \\
\hline & $\begin{array}{l}\text { Evaluación sistemática, pertinente y reproducible de competencias / desempeños en el lugar de tra- } \\
\text { bajo en el programa de postítulo en Radiología }\end{array}$ \\
\hline $\begin{array}{l}\text { Gestión } \\
\text { educacional }\end{array}$ & $\begin{array}{l}\text { Propuesta organizacional para mejorar la gestión de la docencia en el programa de postítulo en } \\
\text { Nefrología }\end{array}$ \\
\hline
\end{tabular}

\section{Nivel 3 Conducta}

En relación a cambios en la práctica docente como la disposición a aplicar y/o a practicar lo aprendido, todos los DPP (100\%) manifestaron que usarían el diseño en seis etapas y el modelo de evaluación de Kirkpatrick. Además, 17 (57\%) DPP desarrollaron un proyecto de intervención en el programa de especialidad (Tabla 5).

\section{Discusión}

Este estudio muestra la positiva evaluación de una experiencia de capacitación en planificación educacional diseñada para un grupo de DPP de la EMUC. Los favorables resultados obtenidos en los tres primeros niveles de Kirkpatrick respaldan la conceptualización epistemológica del diseño propuesto y demostraron la efectividad de la intervención realizada.

Aunque la formación en temas curriculares y planificación educacional constituyen uno de los pilares de la capacitación docente, son escasos los programas desarrollados en comparación a aquellos sobre nuevas metodologías y evaluación de aprendizajes ${ }^{12,36,37}$. En consecuencia, diseñar e implementar este curso fue un gran desafío. La evaluación de necesidades generales y necesidades de los DPP facilitó el enfoque general y el uso de recursos que son generalmente limitados ${ }^{38}$. Mantener la coherencia entre propósito, objetivos, contenidos, metodologías y evaluación sirvió de guía para desarrollar el curso y usarlo como modelo de enseñanza en planificación educacional $^{21,22,28}$. Además, para una enseñanza efectiva fue útil la reflexión incorporada a la autoevaluación y evaluación por pares con retroalimentación frecuente, así como el uso de metodologías innovadoras aplicables a la docencia de postgrado como portafolio, debate y proyecto educacional ${ }^{39}$.

Los resultados del estudio apoyaron el enfoque utilizado. Todos los DPP manifestaron una elevada satisfacción y que recomendarían el curso a otros. 
De igual forma, los aprendizajes objetivos evaluados, las diferencias del pre-post test retrospectivo para todas las habilidades, así como una conducta favorable a aplicar lo aprendido y los proyectos de intervención generados demostraron el impacto de la capacitación.

El diseño metodológico del trabajo no permitió medir efectos en el aprendizaje de los residentes y/o la organización. Para ampliar los resultados se requieren estudios de seguimiento a largo plazo para determinar cambios en las prácticas docentes conducentes a la reformulación de los programas basados en competencias con impacto en la formación de residentes y la acreditación. Sin embargo, pocos programas de capacitación docente han reportado resultados en el Nivel 4 (Resultados) de Kirkpatrick, los que han sido siempre favorables ${ }^{22,23,32}$. Para investigar estos efectos educacionales se han propuesto los estudios controlados y utilizar criterios de calidad basados en la mejor evidencia como la metodología de investigación mixta cuantitativa y cualitativa e indicadores de utilidad ante las dificultades reales para hacer una selección y/o asignación aleatoria y controlar por otras variables ${ }^{40}$.

Las proyecciones de la capacitación para los DPP son amplias: incrementar el número de profesores capacitados, hacer seguimiento, implementar los proyectos de intervención, hacer innovaciones y ampliar la capacitación a otras áreas basados en un nuevo diagnóstico de necesidades ${ }^{41}$. Esto es factible sólo cuando existen condiciones favorables como el apoyo de las autoridades y un soporte institucional sostenido para desarrollar programas de formación exitosos con impacto en los profesores, los residentes y la organización ${ }^{42}$.

La importancia del estudio es mostrar una positiva experiencia de capacitación en planificación educacional para los DPP. La metodología empleada en su diseño y evaluación puede servir de guía para desarrollar intervenciones similares que contribuyan al perfeccionamiento docente y de los programas, con impacto en la formación de los especialistas egresados y las metas de acreditación.

\section{Referencias}

1. Accreditation Council for Graduate Medical Education (ACGME). Disponible en www.acgme.org/acWebsite/ home/home.asp. [Consultado el 29 de mayo de 2011].

2. General Medical Council (GMC). Disponible en www. gmc-uk.org/index.asp . [Consultado el 29 de noviembre 2011].

3. Royal College of Physicians and Surgeons of Canada. The CanMEDSPhysician Competency Framework. Disponible en http://rcpsc.medical.org/canmeds/index. php . [Consultado el 29 de noviembre de 2011].

4. Varkey P, Karlapudi S, Rose S, Nelson R, Warner M. A systems approach for implementing practice-based learning and improvement and systems-based practice in graduate medical education. Acad Med 2009; 84: 3359.

5. Schwarz MR, Wojtczak A. Global minimum essential requirements: A road towards competence-oriented medical education. Institute for International Medical Education (IIME). Disponible en www.iime.org/documents/sv.htm [Consultado el 29 de noviembre de 2011].

6. Accreditation Council for Graduate Medical Education (ACGME). Program Directors \& Coordinators. Disponible en www.acgme.org/acWebsite/navPages/nav_PDcoord.asp . [Consultado el 29 de noviembre de 2011].

7. McLeod PJ, Steinert Y, Meagher T, McLeod A. The ABC of pedagogy for clinical teachers. Med Educ 2003; 37: 638-44.

8. Shumway JM, Harden RM. AMEE Guide No. 25: The assessment of learning outcomes for the competent and reflective physician. Med Teach 2003; 25: 569-84.

9. Hesketh EA, Bagnall G, Buckley EG, Friedman M, Goodall E, Harden RM et al. A framework for developing excellence as a clinical educator. Med Educ 2001; 35: 555-64.

10. Bordage G, Foley R, Goldyn S. Skills and attributes of directors of educational programmes. Med Educ 2000; 34: 206-10.

11. Harris DL, Krause KC, Parish DC, Smith MU. Academic competencies for medical faculty. Fam Med 2007; 39: 343-50.

12. McLean M, Cilliers F, VanWyk J. AMEE Education Guide No 36: Faculty development: yesterday, today and tomorrow. Med Teach 2008; 30: 555-84.

13. Bligh J. Curriculum design revisited. Med Educ 1999; 33: 082-5.

14. Steinert Y, McLeod PJ. From novice to informed educator: the teaching scholars program for educators in the health sciences. Acad Med 2006; 81: 969-74.

15. Heard J, Allen R, Clardy J. Assessing the needs of residency program directors to meet ACGME general competencies. Acad Med 2002; 77: 750.

16. Beeson MS, Gerson LM, Weigand JV, Jwayyed S, Kuhn GJ. Characteristics of Emergency Medicine program directors. Acad Emerg Med 2006; 13: 166-73. 
17. Arora TK, Kaplan BJ. Who are surgery program directors and what do they need? J Surg Ed 2008; 65: 504-11.

18. Kochar MS, Simpson DE, Brown D. Graduate medical education at the medical college of Wisconsin: new initiatives to respond to the changing residency training environment. WMJ 2003; 102: 38-42.

19. Palmer EJ, Tippy PK, Bope ET, Gillanders WR, Pugno PA, Goertz RA, et al. National Institute For Program Director Development (NIPDD): a collaborative pursuit of excellence. Ann Fam Med 2008; 6: 374-5.

20. Snyder S. A program to teach curriculum development to junior faculty. Fam Med 2001; 33: 382-87.

21. Windish DM, Gozu A, Bass EB, Thomas PA, Sisson SD, Howard DM, et al. A ten. Month program in curriculum development for medical educators. 16 years of experience. J Gen Intern Med 2007; 22: 655-61.

22. Gozu A, Windish DM, Knight A, Thomas PA, Kolodner $\mathrm{K}$, Bass EB, et al. Long-term follow up of a 10-month programme in curriculum development for medical educators: a cohort study. Med Educ 2008; 42: 684-92.

23. Triviño X, Sirhan M, Moore P, Montero L. Impacto de un programa de formación en docencia en una escuela de medicina. Rev Med Chile 2011; 139: 1508-15.

24. Kern DE, Thomas PA, Howard DM, Bass EB. Curriculum development for medical education: a six-step approach. Baltimore: Johns Hopkins University Press; 1998.

25. Kaufman D. ABC of Learning and Teaching in Medicine: Applying Educational Theory in Practice. BMJ 2003; 326: $213-16$

26. Harris I. New expectations for professional competence. En: Curry L, Wergin JE, and associates. Educating professionals. Responding to new expectations for competence and accountability. The Jossey-Bass higher and adult education series. San Francisco: Jossey-Bass Publishers; 1993. p. 17-52.

27. Armstrong E, Doyle J, Bennet N. Transformative professional development of physicians as educators: Assessment of a model. Acad Med 2003; 78: 702-8.

28. Cole KA, Barker LR, Kolodner K, Williamson P, Wright SM, Kern DE. Faculty development in teaching skills: an intensive longitudinal model. Acad Med 2004; 79: 46980

29. O'Sullivan P, Cogbill K, McClain T, Reckase M, Clardy J.
Portfolios as a novel approach for residency evaluation. Acad Psychiatry 2002; 26: 173-9.

30. Carraccio C, Englander R. Evaluating competence using a portfolio: A literature review and web-based application to the ACGME competencies. Teach Learn Med 2004; 16: 381-7.

31. Sociedad de Debate, Universidad Nacional Andrés Bello. Debate según formato Karl Popper. Disponible en: http://www.unab.cl/extension/sociedad_debates/ formatos_debates.asp . [Consultado el 29 de noviembre de 2011].

32. Steinert Y, Mann K, Centeno A, Dolmans D, Spencer J, Gelula M, et al. A systematic review of faculty development initiatives designed to improve teaching effectiveness in medical education: BEME Guide No 8. Med Teach 2006; 28: 497-526.

33. Morrison J. ABC of learning and teaching in medicine: evaluation. BMJ 2003; 326: 385-7.

34. Cook DA. Twelve tips for evaluating educational programs. Med Teach 2010; 32: 296-301.

35. McLeod PJ, Steinert Y, Snell L. Use of retrospective pre/ post assessments in faculty development. Med Educ 2008; $42: 543$.

36. Harris I. Perspectives for curriculum renewal in medical education. Acad Med 1993; 68: 484-6.

37. Harris I. Contribution to professional education from the field of curriculum studies: Research and practice with new traditions of investigation. PERQ 1991; 13: 3-13.

38. Lieff S. Evolving curriculum design: A novel framework for continuous, timely, and relevant curriculum adaptation in faculty development. Acad Med 2009; 84: 127-34.

39. Ottawa Conference Miami 2010. Draft consensus statements and recommendations. Criteria for good assessment. Disponible en: http://www.ottawaconference. org/component/content/article/38.html [Consultado el 29 de noviembre de 2011].

40. Prideaux D. Researching the outcomes of educational interventions: a matter of design BMJ 2002; 324: 126-7.

41. Harden RM. Trends and the future of postgraduate medical education. Emerg Med J 2006; 23: 798-802.

42. Triviño X, Sirhan M, Moore P, Reyes C. Formación en educación de los docentes clínicos de medicina. Rev Med Chile 2009; 137: 1516-22. 